\title{
Economia solidária na saúde mental: Iniciativas de geração de renda e trabalho
}

\author{
Solidary economy in mental health: Income and work generation initiatives \\ Economía solidaria en salud mental: Iniciativas de generación de ingresos y trabajo
}

Recebido: 07/06/2021 | Revisado: 13/06/2021 | Aceito: 16/06/2021 | Publicado: 04/07/2021

\author{
Cleide Correia de Oliveira \\ ORCID: https://orcid.org/0000-0001-8135-449X \\ Universidade Regional do Cariri, Brasil \\ E-mail: cleide.correia@urca.br \\ Francisca Laudeci Martins Souza \\ ORCID: https://orcid.org/0000-0003-4211-3233 \\ Universidade Federal do Cariri, Brasil \\ E-mail: laudecimartins@yahoo.com.br \\ Joaquim Rangel Lucio da Penha \\ ORCID: https://orcid.org/0000-0003-0325-3495 \\ Secretaria Municipal de Educação, Brasil \\ E-mail: Rangel.lucio10@gmail.com
}

\begin{abstract}
Resumo
Objetivo: verificar como as estratégias de geração de renda e as oficinas terapêuticas favorecem a cidadania do usuário no processo de inclusão social. Metodologia: Estudo de abordagem exploratória qualitativa. A coleta de dados ocorreu em Centro de Atenção Psicossocial nas cidades Crato e juazeiro do Norte no Ceará. A população em estudo foram 20 profissionais da saúde mental sendo.: psicólogos, enfermeiros, técnicos de enfermagem, assistentes sociais, farmacêutico, terapeuta ocupacional e psicopedagogo. As entrevistas foram realizadas entre os meses de agosto a dezembro de 2018.Utilizou-se um roteiro de entrevista semiestruturada e analisados de maneira descritiva e submetidas a uma metodologia de Análise multimétodo, organizados em uma pasta preparado e processados no software Iramuteq. Resultados: Aplicou-se a métodogia da Classificação Hierárquica Descendente e obtiveram-se 7 classes. A classe 7 tem a maior representação (20\%) do corpus. Na representação fatorial mostra que a classe 3,7 e 6 referem os tipos de atividades que são desenvolvidas. As classes 3, 7 e 6 evidenciaram as oficinas de geração de renda. A c 5, e a 2 destaca-se a importância de incentivar e comercializar os produtos que são produzidos. A classe 4 interligada com a 3 a importância da participação familiar e as classes 1 e 6 o empoderamento. Conclusão: Apontam a necessidade de uma maior participação dos familiares nas atividades $\mathrm{E}$ a reflexão em torno das potencialidades que podem advir da economia solidária e terapia ocupacional, para elucidar e fortalecer novas possibilidades de proposição de trabalhos inclusivos no âmbito da saúde mental.
\end{abstract}

Palavras-chave: Atenção psicossocial; Oficinas terapêuticas; Reabilitação psicossocial.

\begin{abstract}
Objective: to verify how income generation strategies and therapeutic workshops favor user citizenship in the social inclusion process. Methodology: Study with an exploratory qualitative approach. Data collection took place in a Psychosocial Care Center in the cities of Crato and Juazeiro do Norte, Ceará. The study population was 20 mental health professionals: psychologists, nurses, nursing technicians, social workers, pharmacist, occupational therapist and psychopedagogist. The interviews were conducted from August to December 2018. A semi-structured interview script was used, descriptively analyzed and submitted to a Multimethod Analysis methodology, organized in a prepared folder and processed in the Iramuteq software. Results: The Descending Hierarchical Classification method was applied and 7 classes were obtained. Class 7 has the largest representation (20\%) of the corpus. The factorial representation shows that classes 3,7 and 6 refer to the types of activities that are developed. Classes 3, 7 and 6 evidenced the income generation workshops. A c 5 and a 2 highlight the importance of encouraging and marketing the products that are produced. Grade 4 interlinked with grade 3 the importance of family participation and grades 1 and 6 empowerment. Conclusion: They point to the need for greater participation of family members in activities AND reflection on the potential that can come from the solidarity economy and occupational therapy, to elucidate and strengthen new possibilities for proposing inclusive work in the field of mental health.
\end{abstract}

Keywords: Psychosocial care; Therapeutic workshops; Psychosocial rehabilitation.

\section{Resumen}

Objetivo: verificar cómo las estrategias de generación de ingresos y los talleres terapéuticos favorecen la ciudadanía usuaria en el proceso de inclusión social. Metodología: Estudio con abordaje cualitativo exploratorio. La recolección de datos se realizó en un Centro de Atención Psicosocial en las ciudades de Crato y Juazeiro do Norte, Ceará. La 
población de estudio fue de 20 profesionales de la salud mental: psicólogos, enfermeros, técnicos de enfermería, trabajadores sociales, farmacéutico, terapeuta ocupacional y psicopedagogo. Las entrevistas se realizaron de agosto a diciembre de 2018. Se utilizó un guión de entrevista semiestructurado, analizado descriptivamente y sometido a una metodología de Análisis Multimétodo, organizado en carpeta preparada y procesado en el software Iramuteq. Resultados: Se aplicó el método de Clasificación Jerárquica Descendente y se obtuvieron 7 clases. La clase 7 tiene la mayor representación (20\%) del corpus. La representación factorial muestra que las clases 3,7 y 6 se refieren a los tipos de actividades que se desarrollan. Las clases 3, 7 y 6 evidenciaron los talleres de generación de ingresos. A c 5 y a 2 destacan la importancia de fomentar y comercializar los productos que se producen. El $4^{\circ}$ grado relacionó con el $3^{\circ}$ grado la importancia de la participación familiar y el empoderamiento de los grados 1 y 6 . Conclusión: Señalan la necesidad de una mayor participación de los miembros de la familia en las actividades Y la reflexión sobre el potencial que puede provenir de la economía solidaria y la terapia ocupacional, para dilucidar y fortalecer nuevas posibilidades de proponer un trabajo inclusivo en el campo de la salud mental.

Palabras clave: Atención psicosocial; Talleres terapéuticos; Rehabilitación psicosocial.

\section{Introdução}

A Reforma Psiquiátrica surge dentro de uma perspectiva de confrontar o modelo clássico do paradigma da Psiquiatria, enfatizando não só transformações de caráter técnico, mas também teórico, político, cultural e social (Amorim et al., 2017).

A Reforma Psiquiátrica no Brasil tem início, nos anos 80 no contexto da reforma sanitária e da criação do Sistema Único de Saúde SUS pela Constituição de 1988. A Carta de Bauru de 1987 constitui um marco de grande relevância para o movimento auge com a Lei 10.2016 promulgada em 6 de abril de 2001 (Figueiredo, 2019), a lei apresenta uma reformulação no modelo assistencial em saúde mental uma na qual se pretende política mais social que sanitária (Brasil, 2004).

A Reforma Psiquiátrica (RP) no Brasil tem como proposta fundamental a reformulação do modelo de atenção à saúde mental, fazendo a desconstrução do modelo hospitalocêntrico com a política de desinstitucionalização. Conforme (Amorim et al., 2017) faz-se necessária a desconstrução de uma cultura que é majoritária: a que sustenta a violência, a segregação e o aprisionamento da loucura.

O processo de desinstitucionalização consiste na desconstrução de modelo manicomial tem ocorrido por meio da redução de leitos do Sistema Único de Saúde (SUS) em Hospitais Psiquiátricos, como também a criação de dispositivos como os Centros de Atenção Psicossocial (CAPS) e o Programa de Volta para Casa que originou o Auxílio de Reabilitação Psicossocial. Tais instrumentos de efetivação da política constituem-se em estratégias para o fortalecimento da Rede Atenção Psicossocial (RAPS) (Oliveira et al., 2018).

Rede Atenção Psicossocial de acordo com Oliveira et al (2018) tem como fundamento na lógica da reforma psiquiátrica, cm o fortalecimento de Políticas Públicas de Saúde Mental, centrada no modelo de atenção de base territorial, com ênfase no cuidado integral, na promoção da cidadania e da autonomia, tendo o CAPS como um serviço estratégico do seu fortalecimento. Cujo objetivos desse dispositivo de atenção deve acolher o usuário e reinseri-lo no contexto familiar e social, possibilitando o agenciamento de experiências subjetivas e a inclusão social, por meio do acesso ao trabalho e da geração de renda, vinculados aos princípios da economia.

De acordo com a Portaria n 3088/2011MS, a Rede de Atenção Psicossocial-RAPS é constituída por componentes que buscam o cuidado por meio do acolhimento, do acompanhamento contínuo em situações de urgências, promovendo a reabilitação social entre ouros objetivos a organização dos serviços em rede de atenção à saúde regionalizada, com estabelecimento de ações intersetoriais para garantir a integralidade do cuidado (Brasil, 2011).

Esses serviços visavam ser substitutivos das instituições manicomiais com um novo modelo de cuidado integral através do trabalho em equipe interdisciplinar, criando um novo campo com uma proposta de ampliação e diversificação das práticas na saúde mental: o campo da Atenção Psicossocial (Figueiredo, 2019).

Com os movimentos da Reforma Psiquiátrica e da economia solidária tem provocado uma discussão sobre direitos e a inclusão econômica de pessoas excluídas socialmente (Galves et al., 2016). 
A economia solidária no campo da saúde mental representa perspectiva do fortalecimento de uma luta por outro olhar sobre a loucura. A estratégia da inclusão social pelo trabalho na saúde mental está fundamentada nas Lei n. 9.867/1999 e n. 10.216/2001 uma iniciativa do Ministério da Saúde. No entanto a inclusão social pela via da economia solidária foi regulamentada somente em 2005 pelas Portarias Interministerial No. 353/2005 e a Portaria 1169/2005. A primeira autoriza incentivo financeiro para projetos de municípios que contemplem a inclusão social pelo trabalho na esfera da saúde mental. Já a segunda institui o Grupo de Trabalho de Saúde Mental e Economia Solidária por aliança entre Ministérios da Saúde e do Trabalho e Emprego para articular ações, agendas, parcerias (Rodrigues \& Yasui, 2016).

A economia solidária é uma proposta inovadora na geração de renda e trabalho na atenção psicossocial buscando a inclusão social uma forma diferente de produção, fortalecendo o grupo se agredir o meio ambiente, pensando um em um bem comum (Brasil/MTE, 2015).

A aproximação dos serviços de saúde mental à economia solidária não deve se dar unicamente na perspectiva de criação de empreendimentos, transformando as participantes em micro e pequenas empresárias. Proporcionando a construção de novas relações econômicas, sociais e ecológicas construídas e reconstruídas cotidianamente pelos sujeitos no território. Buscando diminuir a divergência entre normal e patológico, proporcionando relações mais sólidas e promissoras buscando a inclusão social (Alves \& Carnut, 2018).

Dentre as oficinas terapêuticas, podem ser realizadas as oficinas de geração de renda, que possibilitam ao usuário, através de suas habilidades, gerar renda, produzindo uma atividade específica. As oficinas terapêuticas devem buscar desenvolver, nos usuários, o exercício da cidadania, apropriado ao conceito de reabilitação, construir práticas de inclusão e respeitar a diversidade, a subjetividade e a capacidade de cada sujeito (Noronha et al., 2016).

Diante do exposto e considerando-se a pertinência das oficinas terapêuticas em iniciativas de geração de trabalho e renda, bem como a importância destas iniciativas estarem pautadas nos princípios da economia solidária, é que o presente estudo teve como objetivo: Verificar como as estratégias de geração de renda e as oficinas terapêuticas favorecem a cidadania do usuário a partir do processo de inclusão social pelo trabalho.

Almejar-se que o estudo venha a estimular novos estudo sobre a temática para o direcionamento e instrumento de ações políticas e práticas no futuro, entendendo a importância das Políticas Públicas de Saúde Mental e de Economia Solidária como estratégia valiosa nesse processo de construção e inter-relação.

\section{Metodologia}

O estudo é do método exploratório descritivo com abordagem qualitativa. A pesquisa qualitativa conforme Yin (2016), estuda os significados a fato das vidas das pessoas, as condições contextuais. As pesquisas exploratórias descritivas tem como objetivo primordial a descrição a caractização das variáveis que se deseja conhecer a partir dos estudos (Köche, 2009). O cenário de estudo ocorreu em dois municípios do estado do Ceará que fazem parte da região do Cariri, as cidades do Crato e juazeiro do Norte.

A coleta de dados ocorreu no Centro de Atenção Psicossocial (CAPS) nas modalidades CAPS AD e CAPS III, nas cidades acima citadas, no serviço de saúde mental que atende pacientes com transtornos mentais severos e persistentes em sua área territorial, em regime de tratamento intensivo, semi-intensivo e não-intensivo. As entrevistas com os profissionais foram realizadas entre os meses de agosto a dezembro de 2018.Os critérios adotados para escolha dos participantes foram: ser profissional do CAPS e estar presente no dia agendado para a entrevista. Foram excluídos os profissionais que estavam afastados do serviço em decorrência de licença ou férias de qualquer natureza.

Os dados foram coletados através de um roteiro de entrevista semiestruturada, dispondo de questões abertas. Os mesmos foram submetidos a uma análise multimétodo. A seguir, organizados em uma pasta de dados preparado 
especificamente para ser processado no software Iramuteq (Interface de R pourles Analyses Multidimensionnelles de Texte set de Questionnaires), a partir do seu corpus. O corpus é construído pelo pesquisador, tratando-se de um conjunto de unidades de contexto inicial que se pretende analisar. O Iramuteq é um software gratuito e desenvolvido na Universidade de Toulouse França, sob a lógica da open source, licenciado por GNU GPL (v2). Este aportar-se no ambiente estatístico do software R e na linguagem python (FILHO et al, 2019).

Um programa de informática com vários tipos de análise de dados textuais, desde as mais simples, como a lexicografia básica (cálculo de frequência de palavras), até análises multivariadas (classificação hierárquica descendente, análises de similitude). O programa faz a organização e a distribuição do vocabulário de forma fácil de compreensão e forma clara (análise de similitude e nuvem de palavras) (Vizeu \& Justo, 2013).

Neste estudo, utiliza-se a nuvem de palavras e a análise de similitude. Dessa forma, as palavras são agrupadas e organizadas graficamente de acordo com a sua frequência, o que possibilita facilmente a sua identificação, a partir de um único arquivo, denominado corpus, que reúne os textos originados pelas entrevistas (Vizeu \& Justo, 2013).

Utilizo-o a Classificação Hierárquica Descendente (CHD) que faz a descrição das classes. Dessa forma, a CHD é baseada no algoritmo proposto para o software Alceste por Reiner e análise léxica, fornece contextos e classes de discursos caracterizados por seus vocabulários. As classes geradas representam o ambiente de sentido das palavras e podem indicar representações sociais ou elementos de representações sociais referentes ao objeto social estudado (Soares et al., 2015).

Com o processamento dos dados, iniciou-se a análise das classes fornecidas pelo software IRAMUTEQ, na qual as Unidades de Contexto Elementar-UCEs agrupadas em cada classe são lidas exaustivamente para compreender e nominar cada uma das classes. Após a etapa de processamento dos dados, iniciou-se a fase de análise de dados, que utilizou como base os normas da abordagem de pesquisa qualitativa, direcionada por seis passos nos quais são (1): passo 1 - organização e preparo dos dados para a análise, etapa realizada para a confecção do corpus; passo 2 - leitura de todos os dados e releituras para avaliação do conteúdo transcrito; passo 3 - análise detalhada com o processo de codificação, realizado no software IRAMUTEQ, que confeccionou o dicionário de palavras; passo 4 - utilização do processo de codificação para descrever as categorias ou temas para análise, com avaliação de todas as classes apresentadas no dendograma; passo 5 - informação de como a descrição e os temas são representados na narrativa qualitativa, fornecidas pelos profissionais do estudo e sustentadas pela literatura consultada após a análise das categorias e passo 6 - extração do significado dos dados, e, após sua análise, apresentação dos resultados pelo pesquisador por meio de figuras e recortes das falas dos participantes do estudo, por meio de sua interpretação pessoal, sustentada na literatura pertinente.

A coleta de dados foi realizada por intermédio de entrevista semiestruturada transcrita na íntegra, após a assinatura do Termo de Consentimento Livre e Esclarecido (TCLE). Os participantes receberam os pseudônimos E1, E2, E3, E4, E5, E6, E7, E8, E9, E10, E11, E12, E13 e E14, de acordo com a sequência em que foram feitas as entrevistas. A avaliação dos dados coletados ocorreu por meio da Análise Temática de Bardin (2011). E seguidas na pesquisa foram: pré-análise, exploração do material e tratamento dos resultados/inferência/interpretação. Foram realizadas as seguintes categorias temáticas:

Esta pesquisa está vinculada a uma investigação mais ampla, intitulada: Cuidar em Saúde: saberes e práticas, desenvolvida na Região Metropolitana do Cariri Ceará, a qual foi submetida à Plataforma Brasil, sendo aprovada pelo Comitê de Ética da Universidade Regional do Cariri, sob Parecer Favorável N².218.604.

\section{Resultados e Discussão}

A pesquisa foi constituída por 20 profissionais. São eles: cinco psicólogos, cinco enfermeiros, cinco técnicos de enfermagem, dois assistentes sociais, um farmacêutico, um terapeuta ocupacional e um psicopedagogo. O perfil identificado revelou uma participação eminentemente feminina com apenas um integrante do sexo masculino, cuja faixa etária varia entre 
25 a 55 anos.

Em relação ao tempo de experiência no âmbito da saúde mental, a maioria afirmou possuir acima de três anos, a exceção de um entrevistado que possui tempo inferior a um ano e seis meses. Cabe ressaltar que a maioria possui pósgraduação lato sensu em diversos campos do conhecimento as quais: Gestão em Saúde, Direito das Famílias, Terapia Intensiva, Saúde Pública, Saúde da Família, Auditoria em Saúde, Enfermagem, Obstetrícia, Serviço Social e Humanização e Saúde Mental).

O corpus foi constituído por 343 Unidades de Contexto Iniciais (UCI) com 42 segmentos analisados, ou seja, 83.33\%\% do corpus, 1216 foi o número de ocorrências e frequências, com a frequência $>=3: 60$, com 262 formas, formas suplementares 32, média das formas por segmentos 28.952381. A partir de matrizes, cruzando segmentos de texto e palavras, aplicou-se o método da Classificação Hierárquica Descendente (CHD) e obtive-se 7 classes como vem representada a Figura 1, abaixo descrevendo as 7 classes com as suas interações.

Figura 1. Dendrograma da Classificação Hierárquica Descendente.

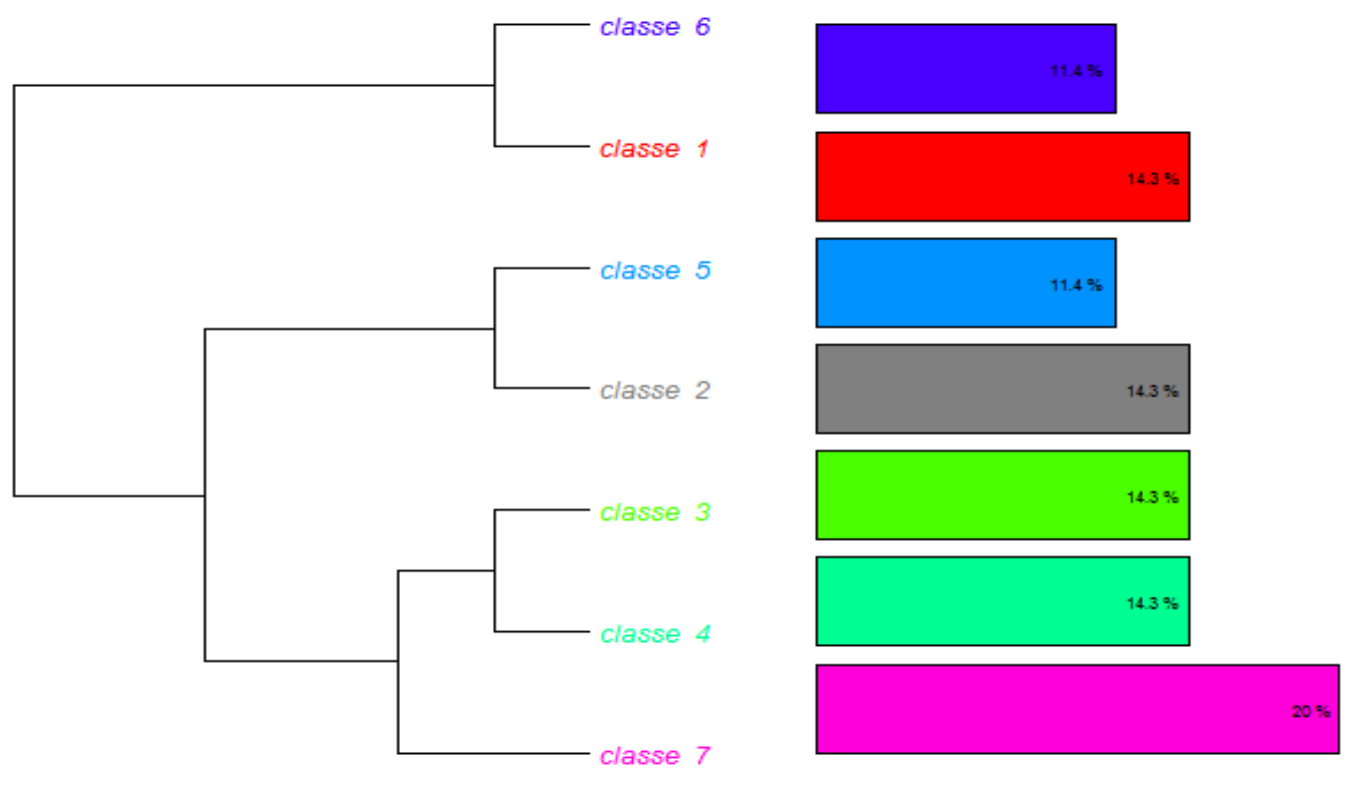

Fontes: Oliveira (2018), Software Iramutec (2018).

O Dendrograma de Classificação Hierárquica Descendente (CHD) 1 (Figura 1) acima permite compreender as expressões e cada uma das palavras proferidas pelos participantes, analisando-as a partir de seus lugares e inserções sociais (Austregesilo, 2015).

Na figura 01, acima ilustra as relações interclasses, a leitura deve ser feita de cima para baixo, ou seja, num primeiro momento, o corpus foi dividido em dois subgrupos. Num segundo momento, o subgrupo superior foi dividido em três, do qual resultaram as classes 6,1 e 7 e o subgrupo inferior foi também dividido em duas classes, a classe 3 e 4 e a classe 7 . Isso significa que as classes 2 e 5 possuem menor relação ou proximidade com as classes 1 e 6 . A classe 3 possui maior relação ou proximidade com a classe 1, assim como a classe 2 possui mais relação ou proximidade com a classe 5 .

Podemos observar na análise do dendrograma (Figura 1) e do plano fatorial (Figura 2) que as classes dois e fortalece a importância do empoderamento e ao incentivo de produzir e comercializar no CAPS através da economia solidária. Há uma interpelação entre as classes. 
Figura 2. Representação Fatorial.

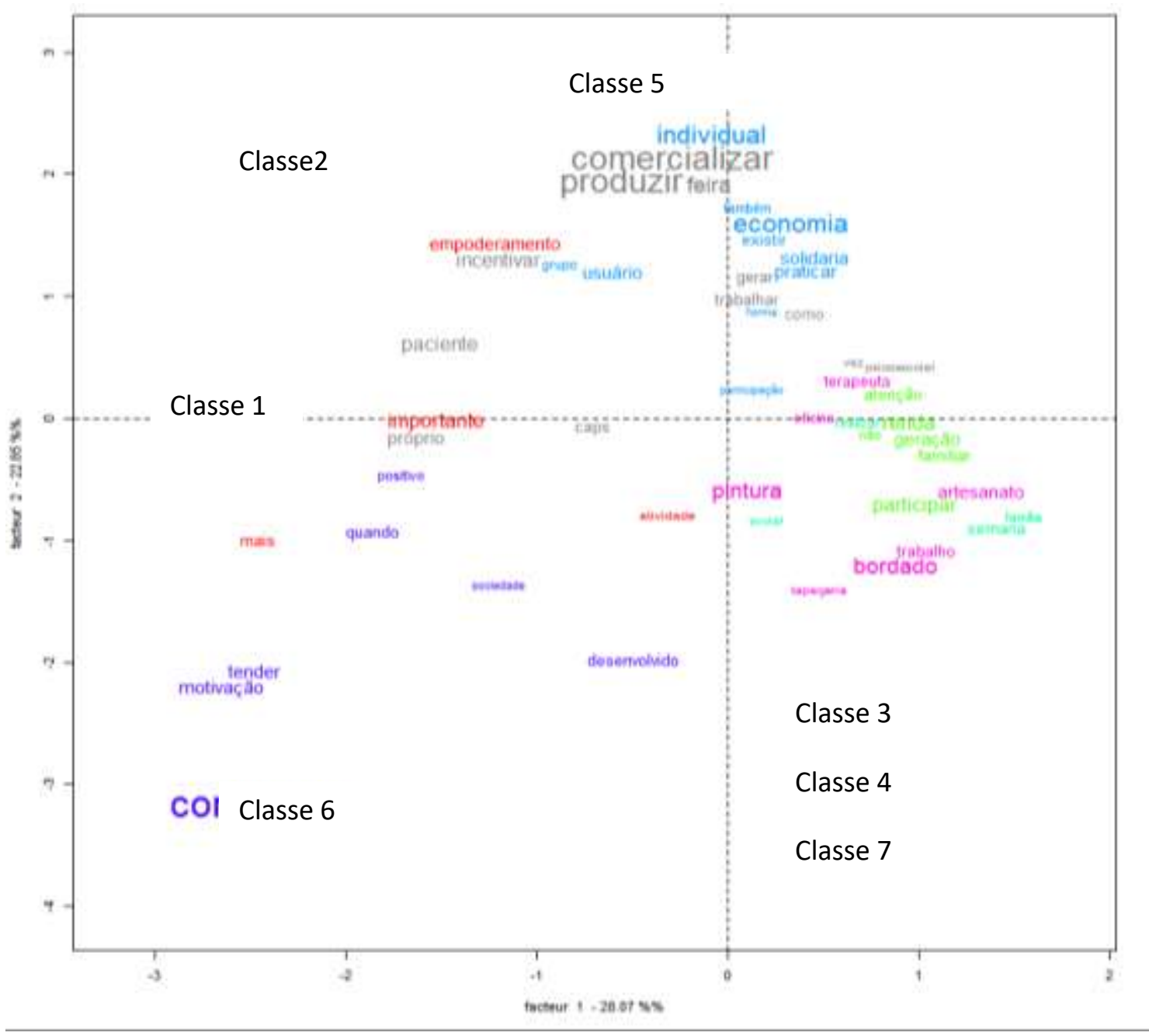

Fontes: Oliveira (2018), Software Iramutec (2018).

Pôde-se perceber, por meio da observação da representação fatorial (Figura 2), que a classe 3,7 e 6 referem os tipos de atividades que são desenvolvidas no CAPS, tais como: pintura, artesanato, bordado, tapeçaria, pintura que é importante os usuários participarem das atividades de oficinas. As classes 3, 7 e 6 aparecem interligadas, o que evidenciou as oficinas de geração de renda. A classe 5, e a 2 destaca-se a importância de incentivar e comercializar os produtos que são produzidos nas oficinas terapêuticas e aparece também o empoderamento para incentivar o usuário do CAPS. A classe 4 interligada com a 3 cita a importância da participação familiar nas atividades no CAPS, já as classes 1 e 6 falam do empoderamento, incentivar os usuários nas atividades. 
Figura 3. Dendrograma das Classes.

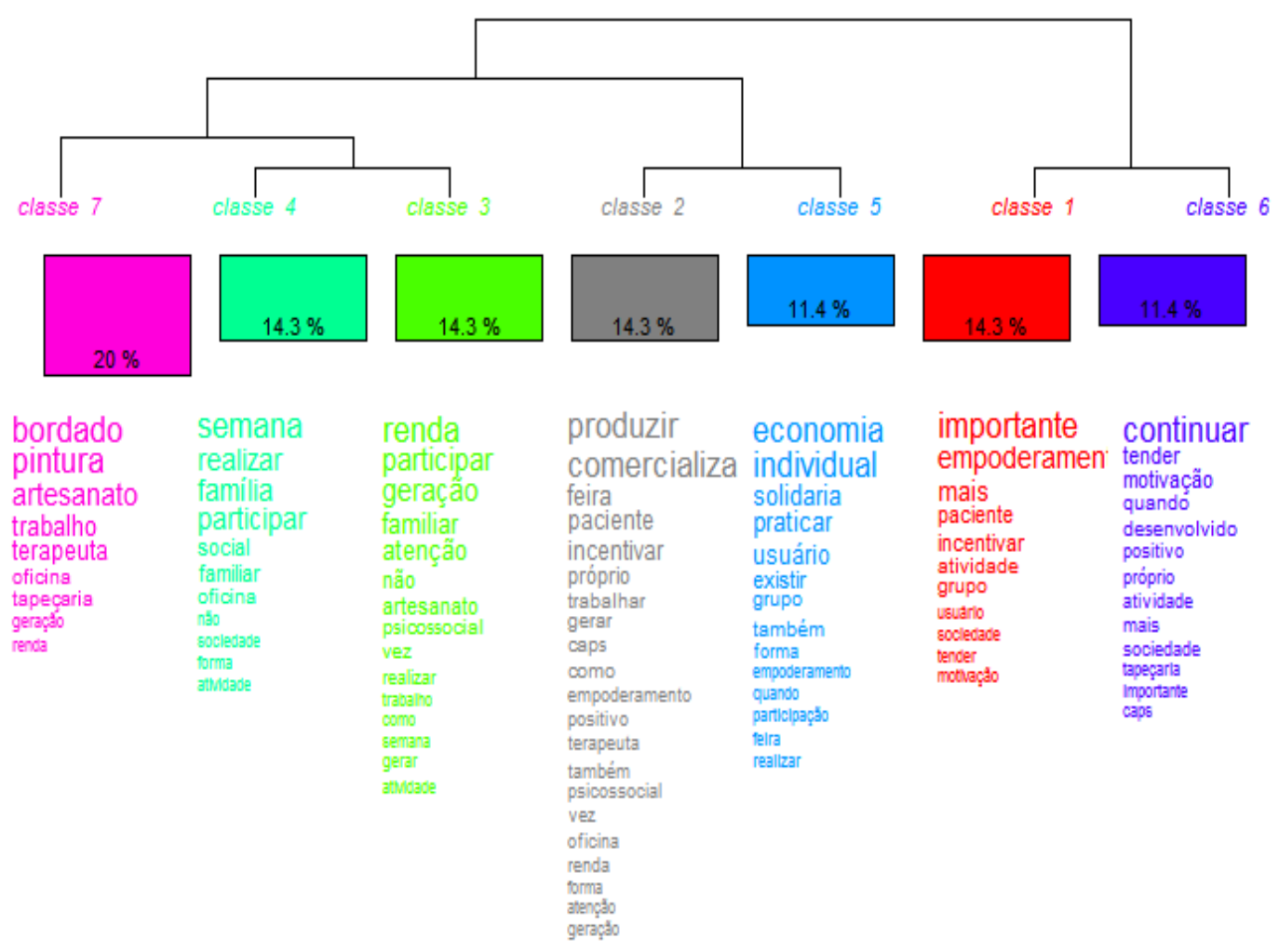

Fontes: Oliveira (2018), Software Iramutec (2018).

Com o dendrograma foi possível visualizar as palavras que obtiveram maior porcentagem quanto à frequência média entre si e diferente entre elas. Esse dicionário de palavras proporcionou, através da utilização do qui-quadrado ( $\chi 2)$, a análise das palavras que apresentaram valor maior que 3,60 e $p<0,0001$.

A Classificação Hierárquica Descendente (CHD) conforme Camargo (2013), qualificou os segmentos de texto em função dos seus respectivos vocabulários, analisou lexicograficamente o texto e categorizou sete classes de respostas associadas oficina de geração de renda, empoderamento a importância da realização das atividades no Centro de Atenção Psicossocial - CAPS.

Do total de 1.216 Unidades de Contexto Inicial (UCI's) presentes no corpus, foram retidas 343 Unidades de Contexto Elementar (UCE's), selecionadas de acordo com os valores do $\left(x^{2}\right)$. Pela leitura das palavras em destaque, e de sua inserção nos segmentos dos textos, foi possível alcançar os objetivos da pesquisa voltada a verificar a ligação estabelecida verificou como as estratégias/oficinas favorecem a cidadania do usuário a partir do processo de inclusão social pelo trabalho e a contribuição dos projetos/oficinas que contribuem para o fortalecimento da Rede de Atenção Psicossocial (RAPS), bem como para o desenvolvimento sustentável da Região Metropolitana do Cariri.

\section{Classe7: estratégias de oficina terapêutica (ot)}

A Classe 7 revelou que as informações dos participantes sobre os tipos de oficinas realizadas no CAPS. Vejamos a seguir: 
As atividades com as oficinas de artesanato, tapeçaria, pintura, bordado teatro, música entre outras atividades, a participação das famílias ainda é pouca (E 2)

Realizadas as oficinas de geração de renda, as oficinas de artesanato com o artesão e a terapeuta. São os trabalhos manuais, pinturas, bordado entre outras atividades (E3).

Não são realizadas oficinas de geração de renda no centro de atenção psicossocial em que trabalho. Somente os trabalhos desenvolvidos com o artesanato, atividades com pintura, bordado e tapeçaria. Temos baixa adesão dos familiares nas atividades do Centro de Atenção Psicossocial (E4).

As atividades com as oficinas de artesanato, tapeçaria, pintura, bordado teatro, música entre outras atividades, a participação das famílias ainda é pouca (E5).

Nos depoimentos acima dos participantes, percebe-se que dentre as estratégias de tratamento oferecidas no CAPS, estão as atividades de Oficinas Terapêuticas (OT), os trabalhos manuais como pintura, bordado e a tapeçaria.

Os CAPSs são espaços de encontros de usuários, familiares e profissionais, assim como de visitantes, profissionais ou pessoas das instituições do território, que promova a circulação de pessoas, a troca de experiência, bate-papos, realização de saraus e outros momentos culturais (BRASIL, 2015).

Os participantes relataram a baixa adesão dos familiares nas atividades. As atividades realizadas em maior número são as pinturas, bordados e tapeçaria. Corroborando com os achados, Ferreira (2018), aponta que quando o cliente de saúde aceita participar de uma oficina, ele se vê como uma pessoa "normal", que pode agradar a seus familiares e participar da sociedade que o excluí. A ideia de inclusão gera maior emancipação, além de fortalecer a sustentabilidade por meio dos produtos gerados pelos usuários no âmbito dos serviços de saúde mental.

O estudo de Pacheco et al. (2018) frisa-se a importância de dar voz aos usuários e aos familiares desses, nos equipamentos de saúde criados a partir da Reforma Psiquiátrica para um tratamento mais qualificado e participativo.

No estudo de Ibiapina et al. (2017), obteve-se resultados semelhantes ao da pesquisa, nos quais ressaltou-se que as atividades de pinturas são as que acontecem com maior frequência no cotidiano do CAPS, pois servem para beneficiar o equilíbrio emocional de cada indivíduo, facilitando a sua expressão e a superação de bloqueios, ocupação da mente tais como os medos a inseguranças e mantendo uma relação mais saudável consigo e com os outros, fortalecendo uma melhor elevação da autoestima.

As atividades de OT no CAPS representam uma importante ferramenta de ressocialização e inserção individual e coletiva, na medida em que possibilita o trabalho, o agir e o pensar coletivo, a partir de uma lógica de respeito à diversidade e à subjetividade e de estímulo à capacidade de cada pessoa (Noronha et al., 2017).

Corroborando com os nossos resultados Ibiapina et al. (2017) refere ainda que as oficinas têm sido entendidas como espaços de produção e manejo de subjetividade, de reconstrução de vínculos entre os sujeitos em sofrimento psíquico e seus grupos sociais, além de irem ao encontro dos discursos de quem cuida e de quem é cuidado.

Apoiando os resultados, Farias et al. (2016) afirmam que as oficinas terapêuticas podem constituir uma importante ferramenta para a produção do paciente e no seu processo de reabilitação psicossocial mais efetivo.

A OT é reconhecida como fator primordial do tratamento do usuário no CAPS, proporcionando a reabilitação, adaptação da sua vida individual como também em grupo, compartilhamento de experiências, levando em consideração a realidade em que estão inseridos (Ibipina et al., 2017).

Essas OT são essenciais para o processo de ressocialização dos indivíduos com transtornos psíquicos são instrumentos indispensáveis, proporcionando-lhes não apenas aptidão para realizar atividades, mas, sobretudo, por exercitá-los e por ajudá- 
los a colocar em prática seus desejos (Monteiro et al, 2017).

No estudo de Ibiapina et al. (2016), ressalta-se a importância da participação dos usuários do CAPS em diversas atividades socio terapêuticas, tais como, as atividades de pinturas, dinâmicas, biscuit, recorte e colagens de desenhos dentro do CAPS com isso têm proporcionando cada dia mais o desenvolvimento de suas habilidades.

Nas entrevistas, os profissionais mencionaram a falta de adesão dos familiares nas atividades de oficinas terapêuticas. Diante do exposto, torna-se importante a participação dos familiares no tratamento dos usuários, para a reinserção social do seu familiar, o acompanhamento do tratamento, melhorar a autoestima e resgate da cidadania. O objetivo da reforma psiquiátrica é restabelecer os direitos e a cidadania dos usuários dos serviços de saúde mental (Brasil, 2011).

\section{Classe 4 e 3 a oficina de geração de renda e trabalho}

No Centro de Atenção Psicossocial são realizadas as oficinas de geração de renda, percebe-se também que a produção oriunda das oficinas é realizada em pequenas feiras e são comercializadas no próprio CAPS ou em praças públicas, as afirmativas refletem nos depoimentos dos participantes.

No Centro de Atenção Psicossocial são realizadas as oficinas de geração de renda, pois são importantes para a recuperação do usuário e a reabilitação psicossocial (E2)

As práticas de economia solidária realizadas no Centro Atenção Psicossocial realizam oficinas de pintura e seus quadros são vendidos em feiras, praças e em eventos ou os funcionários compram os produtos (E5)

Realizamos a prática da oficina solidária a feirinha que é realizada com o que é produzido pelos pacientes é separado e vendido em meses eventuais. É comercializado no CAPS em eventos em faculdades aqui na cidade como também na praça. É organizada a feirinha pela terapeuta ocupacional (E3)

Nos discursos supracitados é reconhecida a importância da oficina terapêutica como fator de grande importância para o tratamento do usuário no CAPS, proporcionando-lhe melhor reabilitação psicossocial e melhoria na autoestima. Pode-se perceber nas falas que as feiras para a comercialização dos produtos realizados pelos usuários do CAPSs ainda são reduzidas e organizada pelo Terapeuta Ocupacional.

Com o avanço da reforma psiquiátrica, da mudança de paradigma e da construção de práticas cujo objetivo é a inclusão social, a geração de trabalho/renda e o acesso do usuário à vida produtiva são tidas como ações primordiais no fortalecimento da autonomia e emancipação do sujeito, dando ao mesmo um novo espaço social (Oliveira et al, 2018).

Os resultados do estudo vão de encontro com os de Ibiapina et al (2019), quando reforçam a importância da oficina terapêutica, vista pelos profissionais como uma ferramenta oportuna para canalizar os pensamentos e valores desse usuário, levando à produção de algo útil para si e para a coletividade a sua volta, podendo levá-lo a um processo de reabilitação psicossocial mais efetivo e traçar um caminho de interesse a essa busca do ser social e atuante.

As atividades de geração de renda ainda se desenham de forma discreta, contudo reforçam as iniciativas de instituir hábitos e ações sustentáveis nos serviços de saúde. A economia solidária reafirma a possibilidade de atividades coletivas com benefícios para todos os envolvidos e o meio ambiente (Ferro et al., 2016; Oliveira et al., 2018).

A ampliação das atividades e espaços de divulgação dos bens e produtos produzidos mostra-se como uma perspectiva de crescimento e aprimoramento dessas iniciativas, colaborando com a inclusão social e reafirmando a cidadania dos usuários. 
A Economia Solidária (ES) traz uma abordagem a respeito de uma nova direção na construção de possibilidades de trabalho, geração de renda e convivência em um meio permeado por valores solidários (Ferro et al., 2016).

Observa-se na fala inicial dos profissionais que existe de forma discreta ações de economia solidária no CAPS e parte dos recursos são destinados para os usuários.

A metade da venda dos produtos vai para os usuários e o restante para reposição dos materiais para novas oficinas. As oficinas são ministradas pelos profissionais do CAPS, professores de artesanato, psicólogos, terapeutas ocupacionais e assistentes sociais. (E 6).

Mostrar que as atividades que são desenvolvidas no CAPS são importantes para eles. Mostrar as escolhas os caminhos. Quando eles percebem a própria condição, a importância do tratamento tende a ser positiva, tem a motivação necessária para continuar com suas atividades e o seu próprio tratamento (E7).

O que é produzido durante as oficinas é comercializado pela terapeuta as vezes na feira no próprio CAPS (E9).

É possível perceber nos relatos a realidade do cotidiano no CAPS sobre a importância de o usuário realizar seu tratamento e participar das atividades que são desenvolvidas. Como também são importantes as iniciativas de geração de renda e trabalho com a possibilidade de troca de valores e reinserção social do sujeito e o exercício da cidadania.

A constituição das oficinas fortalece a criação de vínculos, em uma interação entre os oficineiros e desses com seu entorno social. A geração de trabalho e renda é misturada com a geração de sentido para cada um, no mesmo espaço-tempo da oficina, na intenção de que os participantes sejam cada vez mais autônomos e protagonistas de suas histórias (Rodrigues, 2016). Com a proposta do CAPS de desinstitucionalização os usuários inseridos nas oficinas terapêuticas têm a possibilidade da criação, de expressar seus sentimentos e medos através das atividades realizadas e como isso superar o estigma de louco perigoso. O usuário passa a sentir que faz parte da sociedade e que é um sujeito produtivo.

O processo de desinstitucionalização faz-se necessária, uma postura da equipe respeitando as particularidades do usuário de uma forma holística. A essência de um trabalho em equipe multiprofissional é superar a dificuldade que pode encontrar no período de durante o tratamento. Torna-se possível se houver um olhar diferenciado de cada profissional junto ao usuário procurando solucionar qualquer problema (Ibiapina et al., 2017).

O autor supracitado em seu estudo ressalta que as oficinas terapêuticas têm contribuição para o processo terapêutico produtivo e desenvolvimento integral da capacidade do sujeito, com isso ofertando a possibilidades de eliminar ou minimizar as formas de exclusão na sociedade melhorando a satisfação das necessidades dos usuários de trocas por meio da relação com o outro.

\section{Classe 1 empoderamento na atenção psicossocial}

Foi possível perceber nos relatos das participantes a importância das oficinas terapêuticas para o empoderamento na atenção psicossocial como positivo para resgate da autoestima e sua adesão ao tratamento, na busca pela independência financeira e sua inserção na sociedade, como revelam os depoimentos abaixo:

É importante o empoderamento para a ressocialização do paciente no meio familiar e na sociedade. (E 8).

É importante para usuário as oficinas é uma forma de empoderar o usuário a produz e buscar sua renda (E10)

O empoderamento é incentivado aos pacientes é algo positivo e que gera renda (E9)

Mas é importante haver mais atividade para incentivar o empoderamento nos grupos de pacientes (E11) 
O empoderamento contribui à medida que se tronam mais responsáveis pela sua vida e conseguem tomar suas decisões. (E12)

É o empoderamento do usuário e está ligada diretamente a sua reintegração na sociedade. Importante incentivar os pacientes a participação nas atividades do CAPS (E14).

Por meio dos relatos, percebe-se na manifestação dos profissionais de saúde mental que o empoderamento é essencial a reinserção social do usuário. Dentro da atual política de saúde mental, do Ministério da Saúde no Brasil, faz parte do objetivo dos CAPSs a reinserção social dos usuários pelo acesso ao trabalho.

À singularização do sujeito e o empoderamento do usuário significa, dar voz ao sujeito em tratamento, buscando compreender suas angústias e maximizar suas potencialidades (Pacheco et al., 2018).

O empoderamento emerge nas falas de todos os profissionais como necessário e motivador na autonomia e na busca por melhores condições de vida, destacando o incentivo por parte do dispositivo em saúde na realização das atividades para a geração de renda.

A formação de vínculos entre os usuários e profissionais de saúde fortalece as práticas de cuidar. O posicionamento do usuário como protagonista e corresponsável nos processos de geração de renda capacita o indivíduo para exercer a sua cidadania, viabilizando a inclusão social e o empoderamento do paciente (Freitas et al., 2016).

É importante reconhecer as possibilidades de reconstrução e potencialidades do usuário para um tratamento mais qualificado e participativo. Ao dar espaço para que os mesmos atuem ativamente, o paciente interage de forma mais positiva, o que contribui com a evolução terapêutica e aprimora a comunicação entre todos os envolvidos no processo assistencial (Pacheco et al., 2018).

\section{Classe 6 motivação do usuário do caps na adesão ao tratamento}

A partir dos discursos dos profissionais, foi possível compreender as percepções relativas à importância da participação do usuário quanto ao tratamento e sua motivação na inserção das oficinas terapêuticas. Nessas percepções, observa-se que à medida que o paciente adere o tratamento é essencial uma motivação para manutenção e melhoria do quadro.

A partir do momento que os pacientes aderem ao tratamento e compreendem o que será feito, o empoderamento contribui para sua reinserção social através da economia solidária e da oficina de geração de renda (E 01).

Os discursos fortalecem a concepção de que num processo de ressocialização dos indivíduos com transtornos psíquicos o empoderamento contribui para a sua inserção na sociedade.

De acordo com Monteiro et al. (2017), os profissionais são direcionados a desenvolver atividades com diversos recursos buscando romper com o modelo biomédico, cujo objetivo é reinserir o usuário na sociedade e reabilitá-lo.

$\mathrm{O}$ desenvolvimento de atividades terapêuticas propicia ainda novas experiências aos usuários, apresentando-lhes novas possibilidades e vivências em meio coletivo. A adesão ao tratamento é ampliada devido o usuário sentir-se mais integrado a terapêutica e reconhecer o CAPS como um espaço de vínculos e troca.

A transformação e a elevação da autoestima acontecem à medida em que o usuário consegue sentir-se maior que sua doença, motivando-se a participar das atividades propostas e manifestando por meio das oficinas seus sentimentos e anseios. Aponta-se ainda a motivação na descoberta da sua utilidade e potencialidade para superar o sofrimento e, até mesmo, aprender com ele (Ibiapina et al., 2017). 


\section{Nuvem de palavras e análise de similitude}

Pelo método de nuvem de palavras, que agrupa as palavras e as organiza graficamente em função da sua frequência, a palavra atividade foi a de maior prevalência no corpus - 34 vezes, seguida da palavra oficina - 33 vezes e pela palavra realizar, com 24 vezes (Figura 4).

Percebe-se na figura que as palavras são posicionadas de forma aleatória, contudo as mais frequentes aparecem maiores que as outras, demonstrando, assim, seu destaque no corpus de análise da pesquisa. Para fins deste estudo, após as etapas de processamento, foram interpretados os sentidos das palavras nos discursos dos profissionais, dessa forma a palavra "oficina" surge com sentido de atividade.

Também podem ser visualizadas palavras como atividade, renda, empoderamento, trabalho, participar, familiar, usuário, bordado, incentivar, geração de renda. Pode-se constatar que a nuvem de palavras corrobora os resultados explicitados anteriormente.

Figura 4: Nuvem de palavras e análise de similitude

Na nuvem de palavras no gráfico 4 faz uma organização e agrupamento de palavras de acordo com sua função, o qual possibilita identificar as palavras-chaves tais como atividade, oficina, realizar. Com isso percebemos a importância das atividades realizadas no CAPS, reforçando a importância da realização das atividades no CAPS, fortalecendo o vínculo usuário e instituição no que se propõe a reforma psiquiátrica.

Figura 4. Nuvens de palavras.

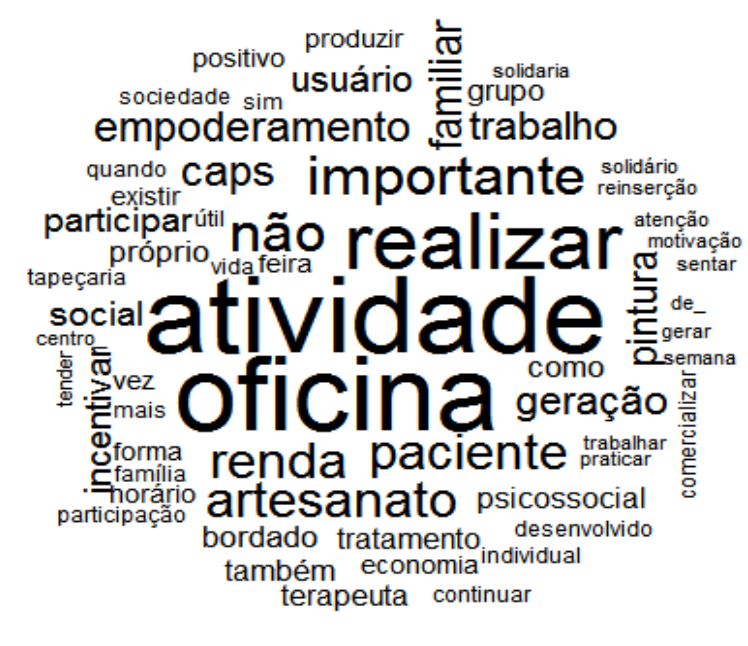

Fontes: Oliveira (2018), Software Iramutec (2018).

A análise de similitudes ancora-se na teoria dos grafos, possibilitando a identificação das ocorrências entre as palavras e seu resultado nas indicações da conexidade entre as mesmas, auxiliando na identificação da estrutura da representação (Marchand; Ratinaud, 2012). A figura 5, abaixo representada pela análise de similitude, vemos nos mostrar o ponto central a oficina de geração de renda, reforçadas pelas palavras atividades, realizar, importante e o empoderamento. Reforçando a importância das atividades terapêuticas nos CAPS.

Figura 5. Analise de similitude. 


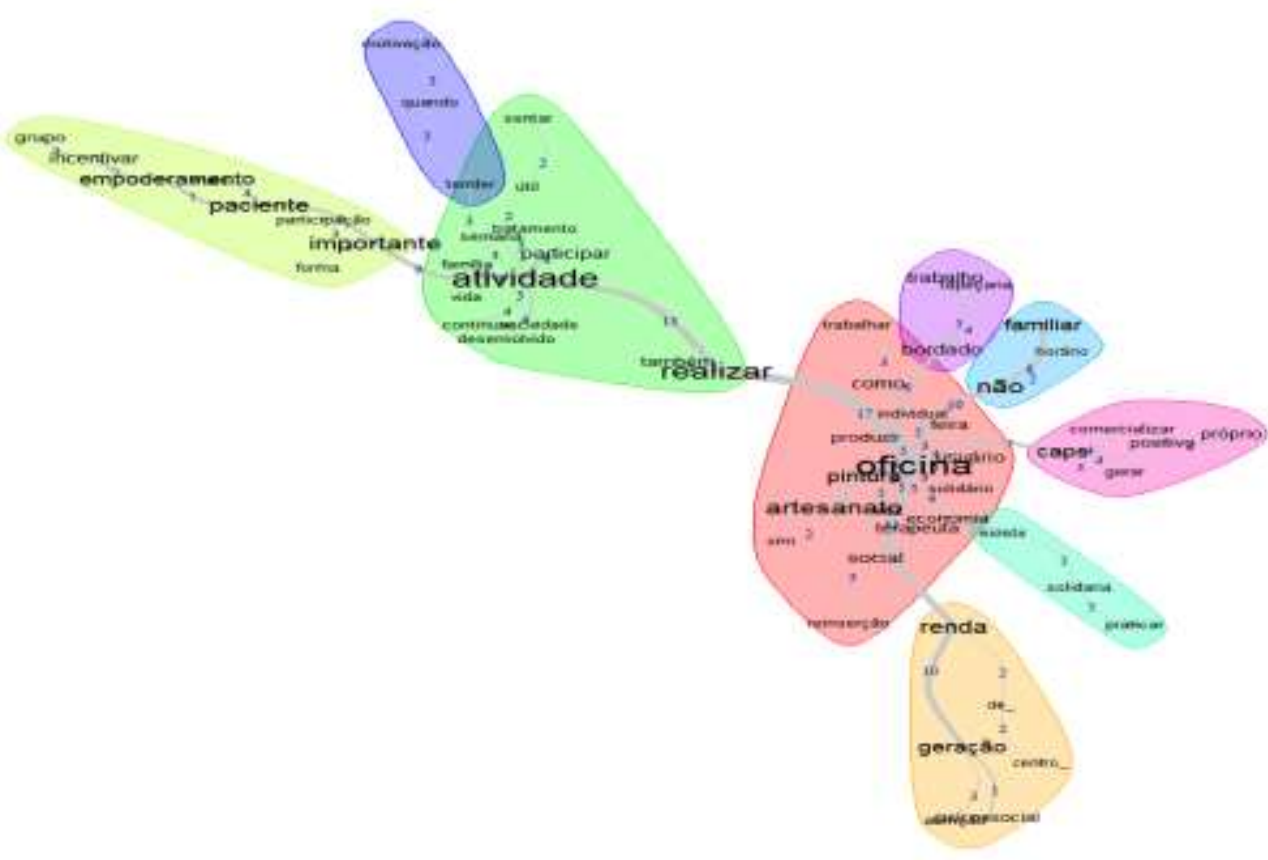

Fontes: Oliveira (2018), Software Iramutec (2018).

A partir da representação gráfica da Figura 5 acima, observa-se que ocorreu um leque semântico de palavras mais frequentes: oficina, empoderamento, realizar, atividade, geração, renda, artesanato.

Os nossos resultados vão de encontro com o estudo de Oliveira et al. (2018), afirmam que os CAPS são serviços abertos vinculados ao Sistema Único de Saúde (SUS), cujo objetivo é acolher o usuário com sofrimento psíquico e reinseri-lo no contexto familiar e comunitário, favorecendo o exercício dos direitos civis, o fortalecimento das relações interpessoais e a inclusão social.

O Projeto Terapêutico Singular (PTS) é uma forma de cuidados ao usuário da Atenção Psicossocial com novas possibilidade de cuidar tais como: as oficinas terapêuticas, atividades comunitárias e artístico-culturais, orientação e acompanhamento do uso de medicação, psicoterapia individual e grupal, visita domiciliar e apoio matricial às equipes da atenção básica.

Vasconcelos et al. (2016), afirmam que o PTS mostra um modo para intensificar o cuidado quando está contextualizada com as dimensões da atenção psicossocial, assumindo postura crítica reflexiva que favoreça o reconhecimento e inclusão de protagonistas neste processo.

Farias et al. (2016) ressaltam a importância do acolhimento do paciente no CAPS, onde é construído o seu Plano Terapêutico Singular (PTS) em conjunto com a equipe de profissionais, incluindo uma ou mais oficinas terapêuticas, com o objetivo de consolidar uma nova possibilidade para que, na condição de usuário do serviço, esse indivíduo possa resgatar sua percepção de saúde e rever seus conceitos de ser ou estar doente ou saudável, estabelecendo estratégias que possam contribuir para a sua qualidade de vida.

\section{Conclusão}

A partir do estudo, verificou-se que as oficinas terapêuticas possibilitam articular e consolidar a política da Reforma Psiquiátrica no Brasil na perspectiva da desinstitucionalização, já que existe interesse e motivação por parte dos profissionais para o trabalho no CAPS, objetivando a transformação do indivíduo em sofrimento psíquico e sua readaptação na sociedade. Observou-se, ainda, que a realização de atividades terapêutica, como a comercialização dos produtos das oficinas 
desenvolvidas pelos profissionais incentiva o empoderamento, e com isso melhora a autoestima e uma maior adesão ao tratamento.

Deste modo, as oficinas terapêuticas são de grande importância para a ressocialização do usuário a sociedade, buscando a construção de vínculo e no resgate da cidadania, socialização, autonomia a autoestima e seu empoderamento na sociedade.

Essa pesquisa apresenta como contribuição às políticas públicas de saúde a evidência que a economia solidária podendo ser fortificada no âmbito do trabalho dos profissionais na Atenção psicossocial, que atuam nesses serviços, para além de um papel estabelecido pelas instituições reguladoras da atuação profissional. Torna-se importante fazer uma reflexão da importância da oficina de geração de renda pelo trabalho, a inclusão do usuário do CAPS no exercício da cidadania autonomia e no seu empoderamento conjuntamente com seus familiares.

Percebeu-se também no estudo uma discreta participação dos familiares dos usuários no cotidiano do CAPS, evidenciando a necessidade da realização de estratégias para inserir o familiar nas atividades. E o incentivo para a participação do tratamento do seu familiar.

Esperamos que estudo venha vislumbrar novas pesquisas e reflexões na área da atenção psicossocial, focando as propostas de reinserção social dos usuários e seus familiares, pois não tendo esgotado as informações sobre essa pesquisa.

\section{Referências}

Alves, S.S., \& Carnut, L. (2018). Trabalho, sentidos e saúde mental: percepção de participantes em um projeto para geração de renda. Semina: Ciências Sociais e Humanas, 39(2), 159-180.

Amorim, A. K. M. A., Dias, M. A., Costa, M. L. F., Araújo, A. C., \& Ferreira, D.S. (2017). Práticas corporais e desinstitucionalização em saúde mental: Desafios e possibilidades. Estudos de Psicologia. Natal. 22(1), 39-49.

BRASIL. (2004). Ministério da Saúde. Saúde Mental no SUS: os Centros de Atenção Psicossocial. Brasília: Ministério da Saúde.

Bardin, L. (2011). Análise de conteúdo. São Paulo: Edição 70.

Brasil. (2004). Ministério da Saúde, Secretaria-Executiva, Secretaria de Atenção à Saúde. Legislação em Saúde Mental: 1990- 2004. 5a ed. Ampliada. Brasília: Ministério da Saúde.

Brasil. (2001). Lei 10.216/2001. A Lei Federal nº 10.216, de abril de 2001.

Brasil. (2015). Ministério da Saúde. Secretaria de Atenção à Saúde. Departamento de Atenção Especializada e Temática. Centros de Atenção Psicossocial e Unidades de Acolhimento como lugares da atenção psicossocial nos territórios: orientações para elaboração de projetos de construção, reforma e ampliação de CAPS e de UA / Ministério da Saúde, Secretaria de Atenção à Saúde, Departamento de Atenção Especializada e Temática. - Brasília: Ministério da Saúde.

Brasil. (2015). Ministério do Trabalho e do Emprego. Economia Solidária. O que é Economia Solidária.

BRASIL. (2011). Portaria No 3.088, de 23 de dezembro de 2011. Institui a Rede de Atenção Psicossocial para pessoas com sofrimento ou transtorno mental e com necessidades decorrentes do uso de crack, álcool e outras drogas, no âmbito do Sistema Único de Saúde (SUS)

Farias, I. D., Thofehrn, M. B., Andrade, A. P. M., Carvalho, L. A., Fernandes, H. N., \& Porto, A. R. (2016). Oficina terapêutica como expressão da subjetividade. SMAD. Revista eletrônica saúde mental álcool e drogas, 12(3), 147-153.

Ferreira, K. F., Carvalho, V. C. S. Oficinas terapêuticas: caminhos de saberes. (2018). Revista da Faculdade de Ciências Médicas de Sorocaba, v2 0i2a5.

Ferro, A. L. F., Mônica, M. A., \& Morgana, B. L. (2015). Economia Solidária, Saúde Mental e a prática do terapeuta ocupacional: relatos de participantes de um grupo de geração de trabalho e renda. Cadernos Brasileiros de Terapia Ocupacional. UFSCar, São Carlos, v. 23, n. 1, p. 101-116, 2015 10.4322/01044931 .

Figueiredo, A. C. (2019). Uma breve revisão da reforma psiquiátrica no Brasil e sua relação com a psicanálise e a psicologia. Revista Psicologia Política, 19(44), 78-87.

Galves, F. R., Luiz, C. C. A., Ramos Júnior, J. E. P., \& Pinho, K. L. R. (2016). Trabalho e geração de renda como produção de cidadania na saúde mental: A experiência do Núcleo de Oficinas e Trabalho de Campinas. Cadernos Brasileiros de Saúde Mental, 8(18), $206-213$.

Freitas, A. C. M., Reckziegel, J. B., \& Barcellos, R. C. (2016). Empoderamento e autonomia em saúde mental: o guia gam como ferramenta de cuidado. Revista Saúde. Santa Maria. v.42, n.2, p.149-156, jul./dez. 2016.

Filho, F. B. N.; Flores, L. C. S., \& Limberger, P. F. (2019). Análise do posicionamento dos restaurantes de São Paulo estrelados pelo guia Michelin com base nas On-line Travel Reviews (OTRS). Revista Brasileira de Pesquisa em Turismo. São Paulo, v. 13, n. 2, p. 1-15. 
Research, Society and Development, v. 10, n. 7, e50610716905, 2021

(CC BY 4.0) | ISSN 2525-3409 | DOI: http://dx.doi.org/10.33448/rsd-v10i7.16905

Ibiapina, A. R. S., Monteiro, C. F. S., Alencar, D. C., Fernandes, M. A., \& Costa Filho, A. A. (2017). Oficinas terapêuticas e as mudanças sociais em portadores de transtorno mental. Revista Esc. Anna Nery. Rio de Janeiro, v. 21, n. 3, e20160375.

Marchand, P., Ratinaud, P. (2011). L'analyse de similitude appliqueé aux corpus textuels: les primaries socialistes pour l'election présidentielle française In: Actes des lleme Journé es internationalesd’Analyse statistique des DonnéesTextuelles. Jadt: Liége, 2012, p. 687-699.

Köche, J.C. (2009). Fundamentos da Metodologia Científica. Editora Vozes $28^{\circ}$ Edição.

Monteiro, C. F. S., Alencar, D. C., Fernandes, M. A., Costa, F., Ibiapina, A. A., \& Ibiapina, A. R. S. (2017). Oficinas terapêuticas e as mudanças sociais em portadores de transtorno mental. Escola Anna Nery, 21(3), e20160375.

Muniz, M. P., Abrahão, A.L., \& Tavares, C. M, M. (2017). O controle da sereia: Trabalho e geração de renda em Saúde Mental. Revista Pró-UniverSUS. 08 (2): 51-57.

Noronha, A. A., Folle, D., Guimarães, A. N, Brum, M. L. B, Schneider, J. F, \& Motta, M. G. C. (2016). Percepções de familiares de adolescentes sobre oficinas terapêuticas em um centro de atenção psicossocial infantil. Revista Gaúcha Enfermagem [Internet].

Oliveira, F. B., Moreira, M. R. C., Lima Júnior, J. F., Nascimento, D. C., Silva, F. M. S., \& Antunes, J. (2018). Articulação das políticas públicas de saúde mental e economia solidária - iniciativas de geração de trabalho e renda. Holos, ano 34 v. 6.

Oliveira, F. B; Lima Junior, J. F., Paiva V. R. DE; Azevedo F, R., \& Macedo M. A. M. (2018). Saúde mental, economia solidária, oficinas de geração de trabalho para usuários em sofrimento psíquico.

Pacheco, S, U, C., Rodrigues, S. R., \& Benatto, M.C. (2018). A importância do empoderamento do usuário de CAPS para a (re)construção do seu projeto de vida. Mental. Barbacena. v. 12, n. 22, p. 72-89.

Santiago, E., \& Yasui, S. (2015). Saúde mental e economia solidária: cartografias do seu discurso político. Psicologia e Sociedade. Belo Horizonte. v. 27, n. 3, p. 700-711.

Rodrigues, A. C., \& Yasui, S. (2016). Oficinas de geração de trabalho e renda na atenção psicossocial: Reflexões sobre um equipamento e suas produções de cuidado. Cadernos brasileiros de saúde mental. Florianópolis, V. 8, N. 20, P. 1-23.

Santos, S., \& Carnut, L. (2018). Trabalho, sentidos e saúde mental: percepção de participantes em um projeto para geração de renda. Semina: Ciências Sociais Humanas. Londrina. v. 39, n. 2, p. 159-180.

Soares R. D, VIllela J. C, Borba L. O, Brusamarello, T, \& Maftum, M. A.(2011). O papel da equipe de enfermagem no Caps. Revista Esc Anna Nery [Internet].15(1):110-5.

Vizeu, C. B., Justo, A. M. (2013). IRAMUTEQ: Um software gratuito para análise de dados textuais. Temas psicol. [Internet].

Veronese, M. V., Gaiger, L. I., \& Ferrarini, A, V. (2017). Sobre a diversidade de formatos e atores sociais no campo da economia solidária. Caderno CRH. Salvador, v. 30, n. 79, p. 89-104.

Vasconcelos, M. G. F., Jorge, M. S. B., Catrib, A. M. F, Bezerra, I. C, \& Franco, T. B. (2016). Therapeutic design in mental health: practices and procedures in dimensions constituents of psychosocial care. Interface (Botucatu). 20(57):313-23.

Yin, R. K. (2016). Pesquisa qualitativa do início ao fim. Porto Alegre. Penso. 